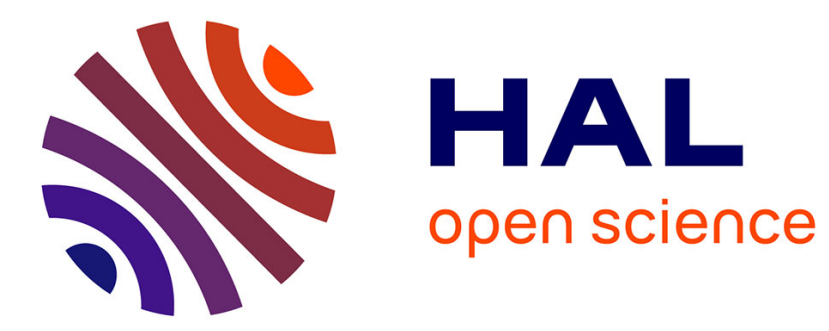

\title{
Dynamic Analysis of Linear and Nonlinear Oscillations of a Beam Under Axial and Transversal Random Poisson Pulses
}

\author{
Marcello Vasta, Angelo Luongo
}

\section{To cite this version:}

Marcello Vasta, Angelo Luongo. Dynamic Analysis of Linear and Nonlinear Oscillations of a Beam Under Axial and Transversal Random Poisson Pulses. Nonlinear Dynamics, 2004, 36 (2-4), pp.421-435. hal-00795111

\author{
HAL Id: hal-00795111 \\ https://hal.science/hal-00795111
}

Submitted on 27 Feb 2013

HAL is a multi-disciplinary open access archive for the deposit and dissemination of scientific research documents, whether they are published or not. The documents may come from teaching and research institutions in France or abroad, or from public or private research centers.
L'archive ouverte pluridisciplinaire $\mathbf{H A L}$, est destinée au dépôt et à la diffusion de documents scientifiques de niveau recherche, publiés ou non, émanant des établissements d'enseignement et de recherche français ou étrangers, des laboratoires publics ou privés. 


\title{
Dynamic Analysis of Linear and Nonlinear Oscillations of a Beam Under Axial and Transversal Random Poisson Pulses
}

\author{
MARCELLO VASTA * and ANGELO LUONGO \\ DISAT, Faculty of Engineering, University of L'Aquila, 67040 Monteluco di Roio, L'Aquila, Italy; "Author for correspondence \\ (e-mail: m.vasta@ing.univaq.it; fax: $+39-0562-434548$ )
}

\begin{abstract}
This paper proposes an approximate explicit probability density function for the analysis of extemal and parametric oscillation of a simply supported beam driven by random pulses. The impulsive loading model adopted is Poisson white noise, which is a process having Dirac delta occurrences with random intensity distributed in time according to Poisson's law. The response probability density function can be obtained by solving the related Kolmogorov-Feller integro-differential equation. An approximate solution is derived by transforming this equation to a first-order partial differential equation. The method of characteristics is then applied to obtain an explicit solution. The theory has been validated through numerical simulations.
\end{abstract}

Key words: external and parametric excitation, Kolmogorov-Feller equation, Poisson random pulses

\section{Introduction}

The problem of estimating the response statistics of nonlinear dynamical systems subjected to random forcing processes has been extensively investigated in the last few decades. In many cases of engineering interest, the structures are subjected to stochastic processes that can be idealized as a train of pulses arriving at random times. Examples of phenomena for which such an idealization is suitable are earthquakes $[1,2]$ and the dynamical behavior of a railway vehicle traveling over an imperfect track [3]. One of the simplest models of impulsive loads is so-called Poisson white noise, which is a process having Dirac delta occurrences with random intensity distributed in time according to Poisson's law. The probabilistic descriptors of the response can be obtained using either the stochastic integrodifferential equation approach [4], or the differential approach [5]. A statistical characterization of the complete response requires a knowledge of the response transition probability density function. For the case of Gaussian white noise input, an approximate transition probability density function can be obtained by solving the well-known Fokker-Planck equation, which is a parabolic partial differential equation. An approximate solution of the Fokker-Planck equation, for small values of the time step, is available in the literature $[6,7]$. This approximate solution has been utilized by many authors for solving engineering problems via the path integral technique [8,9]. For the case of Poisson white noise input, the transition probability density function can be obtained as solution of an integro-differential equation [4], known as the Kolmogorov-Feller equation. A connection has recently been found [10] between the differential approach proposed by Di Paola and Falsone [5] and the integro-differential approach proposed by Gihman and Skorohod [2]. Exact solutions for some particular classes of nonlinear systems excited by both external and parametric delta-correlated excitations have been recently obtained $[11,12]$. A direct numerical solution of the Kolmogorov-Feller equation is not an entirely straightforward matter, even for two-dimensional problems, and only approximate methods have been derived [13-15]. A technique for deriving an approximate response transition probability density function for a 
one-degree of-freedom system, solving the related Kolmogorov-Feller equation, has already been proposed in [16].

In this paper an approximate explicit response probability density function of a beam under external and axial impulsive random Poisson excitation is obtained as a solution of the Kolmogorov-Feller equation. The main difference between external and parametric Poisson white noise consists in the presence of a series of correction terms to take advantage of the non-anticipating property between increments of compound Poisson processes and the response for parametric excitation [5]. However, if in linear or nonlinear oscillators the multiplicative term appears in displacements, this does not produce the need for correction terms. The proposed approach considers parametric excitation that is multiplicative in displacement because the integral term appearing in the differential equation in terms of probability density function (the well known Kolmogorov-Feller equation) offers a better opportunity to find approximate solutions through appropriate physical and mathematical considerations than in the case of multiplicative terms in velocity, where an infinite series of terms appears. The procedure followed consists first in the reduction of the Kolmogorov-Feller equation to a firstorder partial differential equation, using physical considerations about the behavior of the response for small values of the time step. Then the well-known Method of Characteristics is applied to obtain the required solution. The correctness of the solution has been validated through Monte Carlo simulation.

\section{Beam Under Transversal Poisson Pulses}

Let us consider a simply supported beam subjected to a train of stationary Poisson pulses at mid-span defined by

$$
\xi(t)=\sum_{k=1}^{N(t)} A_{k} \delta\left(t-t_{k}\right),
$$

where $\delta(\cdot)$ stands for a Dirac delta. In Equation (1) the random instants $t_{k}$ are independent of the intensities of the spikes $A_{k}$, having assigned probability density function $p_{A}(a)$ and assumed to be independent random variables, while $N(t)$ is an homogeneous Poisson counting process giving the total number of impulses occurring in the time interval $[0, t)$ with mean arrival rate equal to $\lambda$. The equation of motion of the beam can be written as follows:

$$
E J \frac{\partial^{4} w}{\partial x^{4}}+\mu \frac{\partial^{2} w}{\partial t^{2}}+\rho \frac{\partial w}{\partial t}=\xi(t) \delta(x-l / 2)
$$

$w(x, t)$ being the vertical displacement of the beam. In Equation (2) $\rho$ is the beam density; $J$ is the moment of inertia of the beam cross section; $E$ is Young's modulus; $\mu$ is a damping factor; $l$ is the length of the beam.

It is well known that for the simply supported beam, the eigenfunctions are given by the formula

$$
\phi_{n}(x)=\sin \left(\frac{\pi n}{l} x\right)
$$


Solution of Equation (2) can then be obtained by mode superposition

$$
w(x, t)=\sum_{n=1}^{\infty} \phi_{n}(x) Y_{n}(t)
$$

and using the well-known orthogonality properties of the eigenfunction basis, the following set of stochastic uncoupled linear set of differential equations is obtained

$$
\ddot{Y}_{n}+2 D \omega_{n} \dot{Y}_{n}+\omega_{n}^{2} Y_{n}=P_{n} \xi(t), \quad n=1,2, \ldots
$$

where

$$
\omega_{n}=\left(\frac{\pi n}{l}\right)^{2} \sqrt{\frac{E J}{\mu}}, \quad \frac{\rho}{\mu}=2 D \omega_{n} .
$$

For $n=1$ the following single-degree-of-freedom equation of motion is obtained

$$
\ddot{Y}+2 D \omega_{1} \dot{Y}+\omega_{1}^{2} Y=\xi(t) .
$$

The probabilistic characterization of the response can be achieved, when truncating at the first mode, by considering the time evolution of the transition probability of the response

$$
p\left(y, \dot{y} ; t+\tau / y^{\prime}, \dot{y}^{\prime} ; t\right)=\frac{p\left(y, \dot{y}, t+\tau ; y^{\prime}, \dot{y}^{\prime}, t\right)}{p\left(y^{\prime}, \dot{y}^{\prime}, t\right)}
$$

that respects the Kolmogorov-Feller integro-differential equation

$$
\begin{aligned}
\frac{\partial p}{\partial \tau}= & 2 D \omega_{1} \frac{\partial(\dot{y} p)}{\partial \dot{y}}+\omega_{1}^{2} y \frac{\partial p}{\partial \dot{y}}-\dot{y} \frac{\partial p}{\partial y} \\
& +\lambda \int_{\mathbf{a}} p\left(y, \dot{y}-a ; t+\tau / y^{\prime}, \dot{y}^{\prime} ; t\right) p_{A}(a) d a-\lambda p .
\end{aligned}
$$

Equation (9) must be solved subject to the initial condition

$$
p\left(y, \dot{y} ; t / y^{\prime}, \dot{y}^{\prime} ; t\right)=\delta\left(y-y^{\prime}\right) \delta\left(\dot{y}-\dot{y}^{\prime}\right) .
$$

Exact stationary solutions (i.e., $\partial p / \partial t=0$ ) of Equation (9) have been obtained for particular classes of nonlinear systems $[11,12]$. In order to find an approximate solution of Equation (9), because of Equation (10), for small values of $\tau$ the following approximation holds

$$
p\left(y, \dot{y}-a ; t+\tau / y^{\prime}, \dot{y}^{\prime} ; t\right)=\delta\left(y-y^{\prime}\right) \delta\left(\dot{y}-a-\dot{y}^{\prime}\right)+O(\tau) .
$$

where $O(\tau)$ goes to zero as $\tau \rightarrow 0$. Substituing Equation (11) in Equation (9) we obtain

$$
\begin{aligned}
\frac{\partial p}{\partial \tau}= & 2 D \omega_{1} \frac{\partial(\dot{y} p)}{\partial \dot{y}}+\omega_{1}^{2} y \frac{\partial p}{\partial \dot{y}}-\dot{y} \frac{\partial p}{\partial y} \\
& +\lambda \int_{\mathbf{a}} \delta\left(y-y^{\prime}\right) \delta\left(\dot{y}-a-\dot{y}^{\prime}\right) p_{A}(a) d a-\lambda p .
\end{aligned}
$$


Multiplying both members of Equation (12) by the joint probability distribution and then integrating with respect to the initial variables, one obtains the following differential equation for the joint probability density function $p_{t+\tau}=p(y, \dot{y} ; t+\tau)$ corrected up to the order $\tau$ :

$$
\frac{\partial p_{t+\tau}}{\partial \tau}=2 D \omega_{1} \frac{\partial\left(\dot{y} p_{t+\tau}\right)}{\partial \dot{y}}+\omega_{1}^{2} y \frac{\partial p_{t+\tau}}{\partial \dot{y}}-\dot{y} \frac{\partial p_{t+\tau}}{\partial y}+G_{t}(y, \dot{y} ; t)-\lambda p_{t+\tau},
$$

where

$$
G_{t}(y, \dot{y} ; t)=\lambda \int_{\mathbf{a}} p_{t}(y, \dot{y}-a ; t) p_{A}(a) d a
$$

Equation (13) is a linear partial differential equation that can be solved by the Method of Characteristics [17]. In order to construct the solution surface one solves the following system of ordinary differential equations on $s$

$$
\left\{\begin{array}{l}
\frac{d \tau}{d s}=1 \\
\frac{d y}{d s}=\dot{y} \\
\frac{d \dot{y}}{d s}=-\left(2 D \omega_{1} \dot{y}+\omega_{1}^{2} y\right) \\
\frac{d p}{d s}=G_{t}(y, \dot{y} ; t)+\left(2 D \omega_{1}-\lambda\right) p
\end{array}\right.
$$

subject to the following initial conditions

$$
\left\{\begin{array}{l}
\tau(0)=0 \\
y(0)=y^{\prime} \\
\dot{y}(0)=\dot{y}^{\prime} \\
p(0)=p_{t}\left(y^{\prime}, \dot{y}^{\prime} ; t\right) .
\end{array}\right.
$$

Solution of Equation (15) takes the form

$$
\left\{\begin{array}{l}
\tau=s+C_{1} \\
y(s)=C_{2} e^{-D \omega_{1} s} \cos \left(\bar{\omega}_{1} s\right)+C_{3} e^{-D \omega_{1} s} \sin \left(\bar{\omega}_{1} s\right) \\
p(s)=p(0) \exp (c s)+G_{t}(y, \dot{y} ; t) \tau,
\end{array}\right.
$$

where

$$
\bar{\omega}_{1}=\omega_{1} \sqrt{1-D^{2}}, \quad c=2 D \omega_{1}-\lambda,
$$

and because of the initial conditions Equation (16)

$$
C_{1}=0, \quad C_{2}=y^{\prime}, \quad C_{3}=\frac{\dot{y}^{\prime}+D \omega_{1} y^{\prime}}{\bar{\omega}_{1}} .
$$

The solution found is finally

$$
p(y, \dot{y}, t+\tau)=e^{c \tau} p_{t}\left(y^{\prime}(y, \dot{y}), \dot{y}^{\prime}(y, \dot{y}) ; t\right)+G_{t}(y, \dot{y} ; t) \tau,
$$




$$
\left\{\begin{array}{l}
y^{\prime}(y, \dot{y})=\frac{e^{D \omega_{1} \tau}}{\bar{\omega}_{1}}\left[\left(\bar{\omega}_{1} \cos \left(\bar{\omega}_{1} \tau\right)-D \omega_{1} \sin \left(\bar{\omega}_{1} \tau\right)\right) y-\sin \left(\bar{\omega}_{1} \tau\right) \dot{y}\right] \\
\dot{y}^{\prime}(y, \dot{y})=\frac{e^{D \omega_{1} \tau}}{\bar{\omega}_{1}}\left[\omega^{2} \sin \left(\bar{\omega}_{1} \tau\right) y+\left(\bar{\omega}_{1} \cos \left(\bar{\omega}_{1} \tau\right)+D \omega_{1} \sin \left(\bar{\omega}_{1} \tau\right)\right) \dot{y}\right]
\end{array}\right.
$$

It should be noted that the solution proposed in Equation (20) is confined to the probabilistic characterization for the case of $\tau \rightarrow 0$. By increasing the value of $\tau$ the solution (20) needs to consider higher-order terms in power of $\tau$, which have been neglected in the solution procedure.

\section{Beam Under Transversal and Axial Poisson Pulses}

Let us now consider a simply supported beam subjected to a transversal harmonic external load and to a train of stationary axial Poisson pulses, as in Equation (1). The equation of motion of the beam can be written as follows:

$$
E J \frac{\partial^{4} w}{\partial x^{4}}+\mu \frac{\partial^{2} w}{\partial t^{2}}+\rho \frac{\partial w}{\partial t}+\xi(t) \frac{\partial^{2} w}{\partial x^{2}}=\sin (\Omega t),
$$

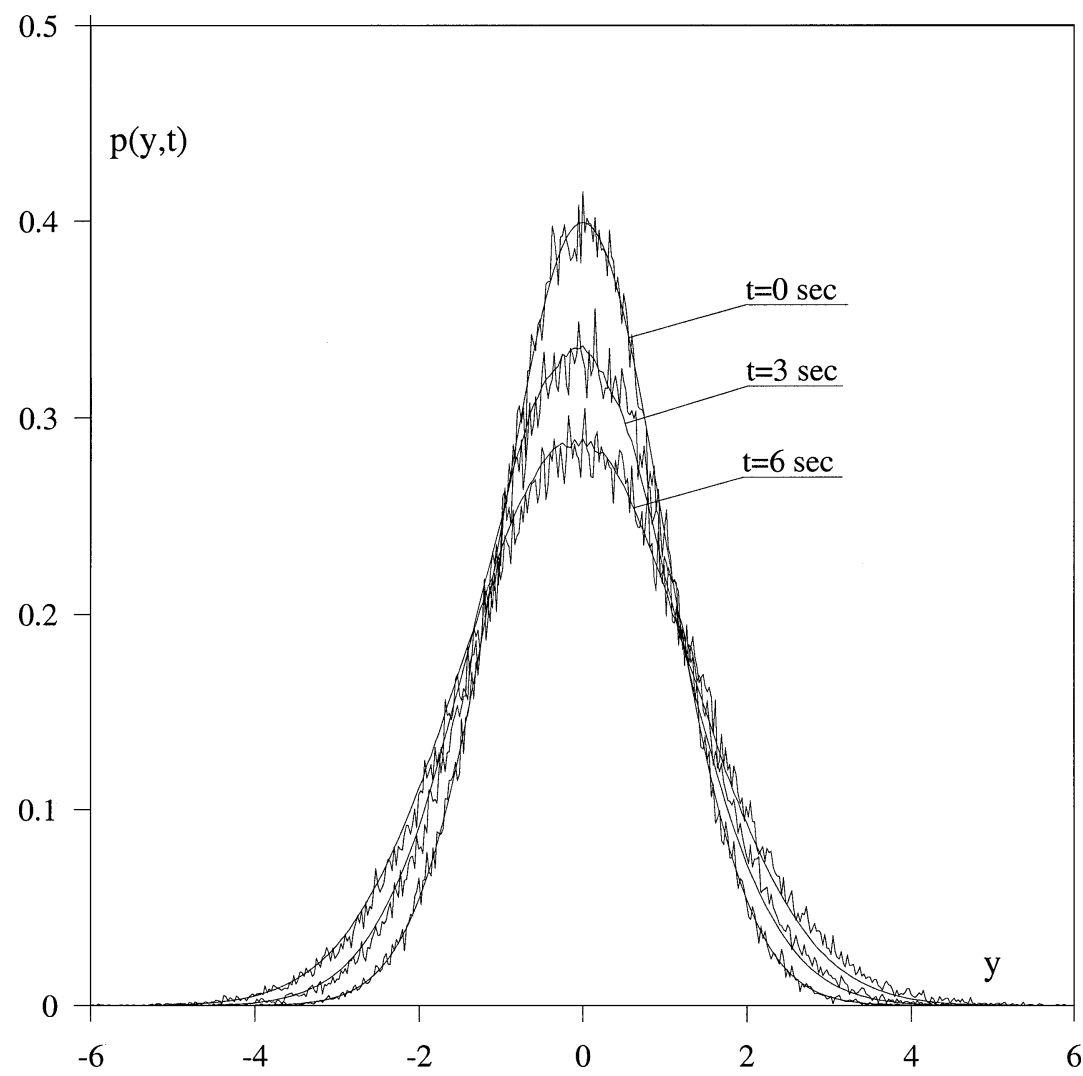

Figure 1. Comparison between analytical solution and simulation for the response probability density function at $t=0,3,6 \mathrm{~s}$. 


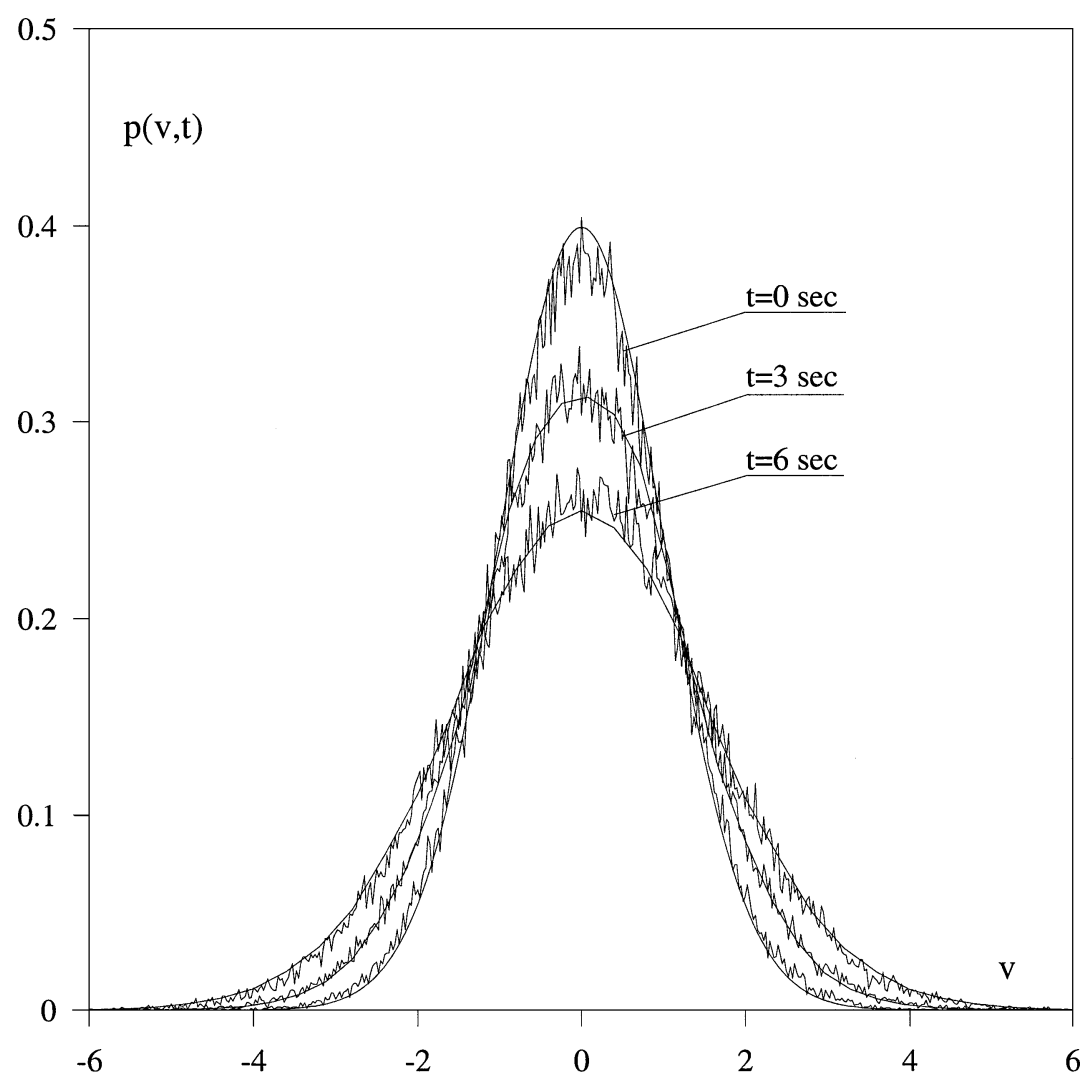

Figure 2. Comparison between analytical solution and simulation for the velocity probability density function at $t=0,3,6 \mathrm{~s}$.

$w(x, t)$ being the vertical displacement of the beam. In Equation (2) $\rho$ is the beam density; $J$ is the moment of inertia of the beam cross section; $E$ is Young's modulus, $\mu$ is a damping factor. Using the mode superposition representation as described in Equations (3-4), and because of the well-known orthogonality properties of the eigenfunction basis, the following set of uncoupled quasilinear equations is obtained

$$
\ddot{Y}_{n}+2 D \omega_{n} \dot{y}_{n}+\omega_{n}^{2}\left[1-\varepsilon_{n} \xi(t)\right] Y_{n}=P_{n} \sin (\Omega t), \quad n=1,2, \ldots
$$

in which

$$
\omega_{n}=\left(\frac{\pi n}{l}\right)^{2} \sqrt{\frac{E J}{\mu}}, \quad \frac{\rho}{\mu}=2 D \omega_{n}, \quad \varepsilon_{n}=\frac{1}{E J}\left(\frac{\pi n}{l}\right)^{2} .
$$

For $n=1$ one obtains the following single-degree-of-freedom equation of motion, of so-called quasilinear type:

$$
\ddot{Y}+2 D \omega_{1} \dot{y}+\omega_{1}^{2}\left[1-\varepsilon_{1} \xi(t)\right] Y=\sin (\Omega t) .
$$




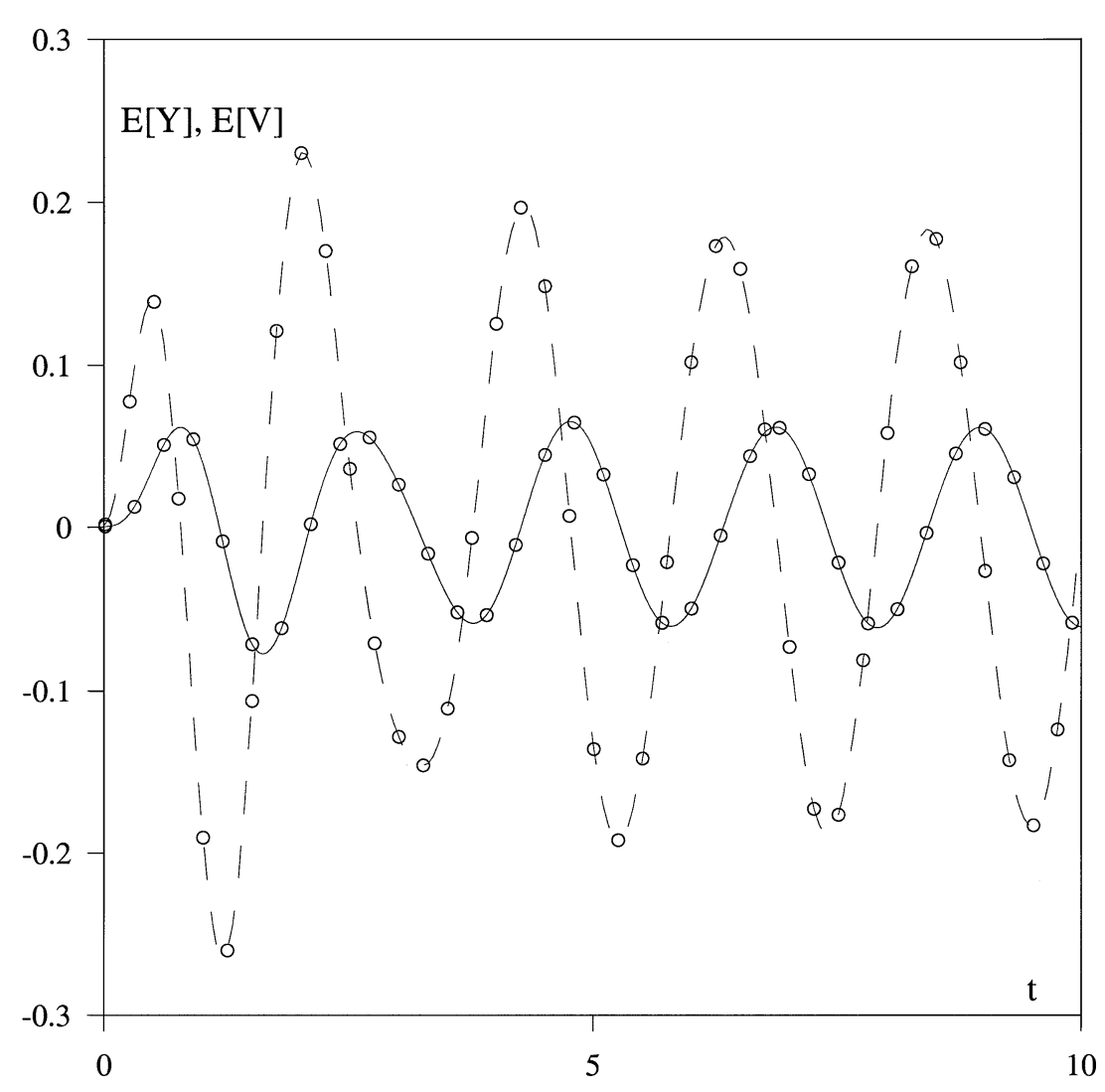

Figure 3. Comparison between analytical solution and simulation for the mean of displacement $E[X]$ and velocity $E[V]$. Continuous line $-E[\mathrm{X}]$, dashed line $-E[V]$, dotted line - simulation.

The probabilistic characterization of the response can be achieved, when truncating at the first mode, by considering the time evolution of the transition probability of the response

$$
p\left(y, \dot{y} ; t+\tau / y^{\prime}, \dot{y}^{\prime} ; t\right)=\frac{p\left(y, \dot{y}, t+\tau ; y^{\prime}, \dot{y}^{\prime}, t\right)}{p\left(y^{\prime}, \dot{y}^{\prime}, t\right)}
$$

that respects the Kolmogorov-Feller integro-differential equation

$$
\begin{aligned}
\frac{\partial p}{\partial \tau}= & 2 D \omega_{1} \frac{\partial(\dot{y} p)}{\partial \dot{y}}+\left(\omega_{1}^{2} y+\sin (\Omega t)\right) \frac{\partial p}{\partial \dot{y}}-\dot{y} \frac{\partial p}{\partial y} \\
& +\lambda \int_{\mathbf{a}} p\left(y, \dot{y}-\omega_{1}^{2} \varepsilon y a ; t+\tau / y^{\prime}, \dot{y}^{\prime} ; t\right) p_{A}(a) d a-\lambda p .
\end{aligned}
$$

Equation (27) must be solved subject to the initial condition, Equation (10). For the case $P_{n}=0$, that is, when only an axial parametric load is considered, solution techniques of Equation (27) in the frequency domain in terms of characteristic function were reported in [18]. By the same considerations outlined in the previous section, Equations (10-12), it can be easily shown that, for small values of $\tau$ the following approximation holds for the evolutionary joint probability density function $p_{t+\tau}=p(y, \dot{y} ; t+\tau)$ :

$$
\frac{\partial p_{t+\tau}}{\partial \tau}=2 D \omega_{1} \frac{\partial\left(\dot{y} p_{t+\tau}\right)}{\partial \dot{y}}+\left(\omega_{1}^{2} y+\sin (\Omega t)\right) \frac{\partial p_{t+\tau}}{\partial \dot{y}}-\dot{y} \frac{\partial p_{t+\tau}}{\partial y}+G_{t}(y, \dot{y} ; t)-\lambda p_{t+\tau}
$$




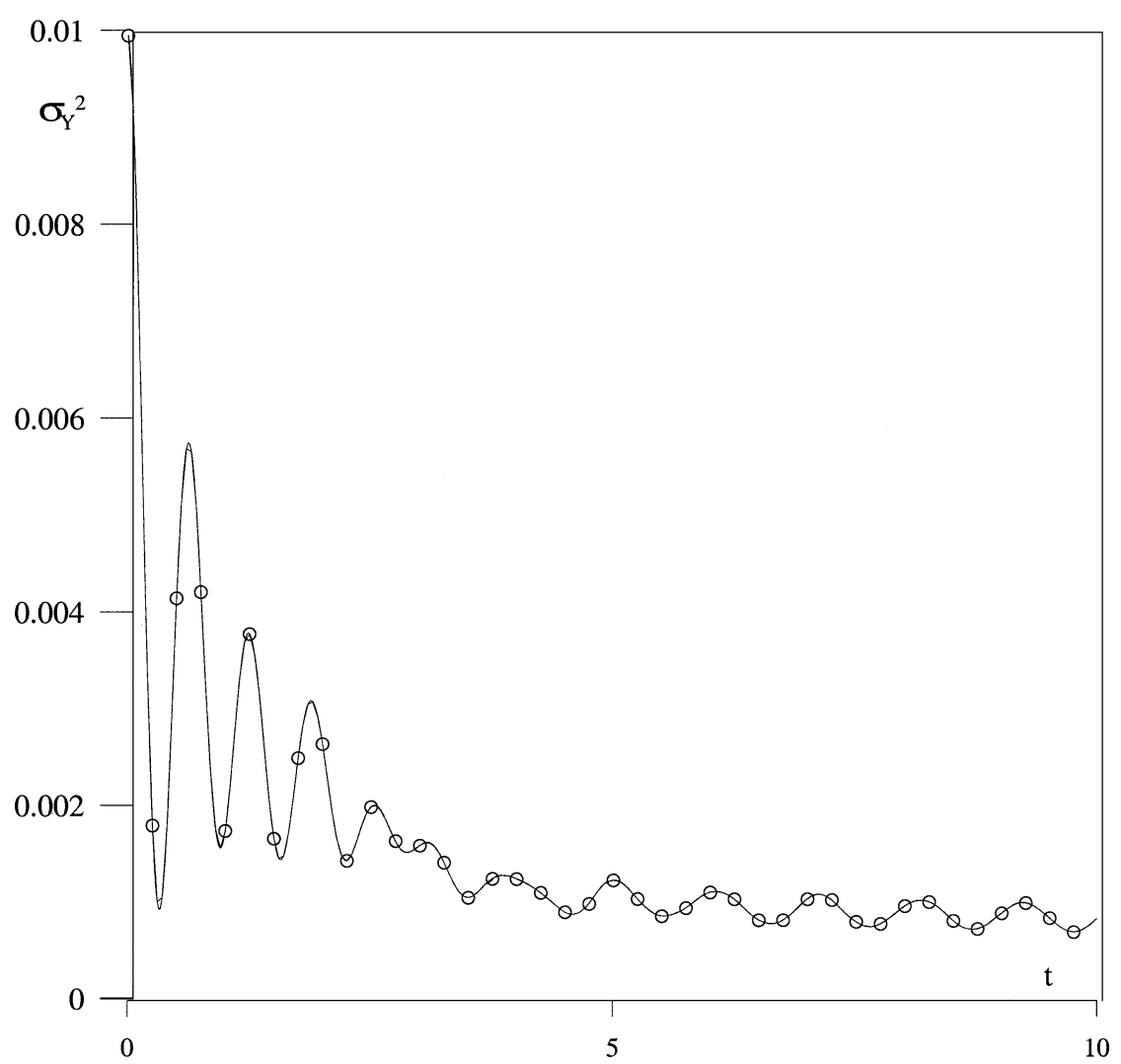

Figure 4. Comparison between analytical solution and simulation for the displacement variance, continuous line $-\sigma_{y}^{2}$, dotted line - simulation.

where

$$
G_{t}(y, \dot{y} ; t)=\lambda \int_{\mathbf{a}} p_{t}\left(y, \dot{y}-\omega_{1}^{2} \varepsilon y a ; t\right) p_{A}(a) d a .
$$

Equation (28) is a linear partial differential equation that can be solved by the Method of Characteristics. In order to construct the solution surface one solves the following system of ordinary differential equations on $s$

$$
\left\{\begin{array}{l}
\frac{d \tau}{d s}=1 \\
\frac{d y}{d s}=\dot{y} \\
\frac{d \dot{y}}{d s}=-\left(2 D \omega_{1} \dot{y}+\omega_{1}^{2} y+\sin (\Omega t)\right) \\
\frac{d p}{d s}=G_{t}(y, \dot{y} ; t)+\left(2 D \omega_{1}-\lambda\right) p
\end{array}\right.
$$




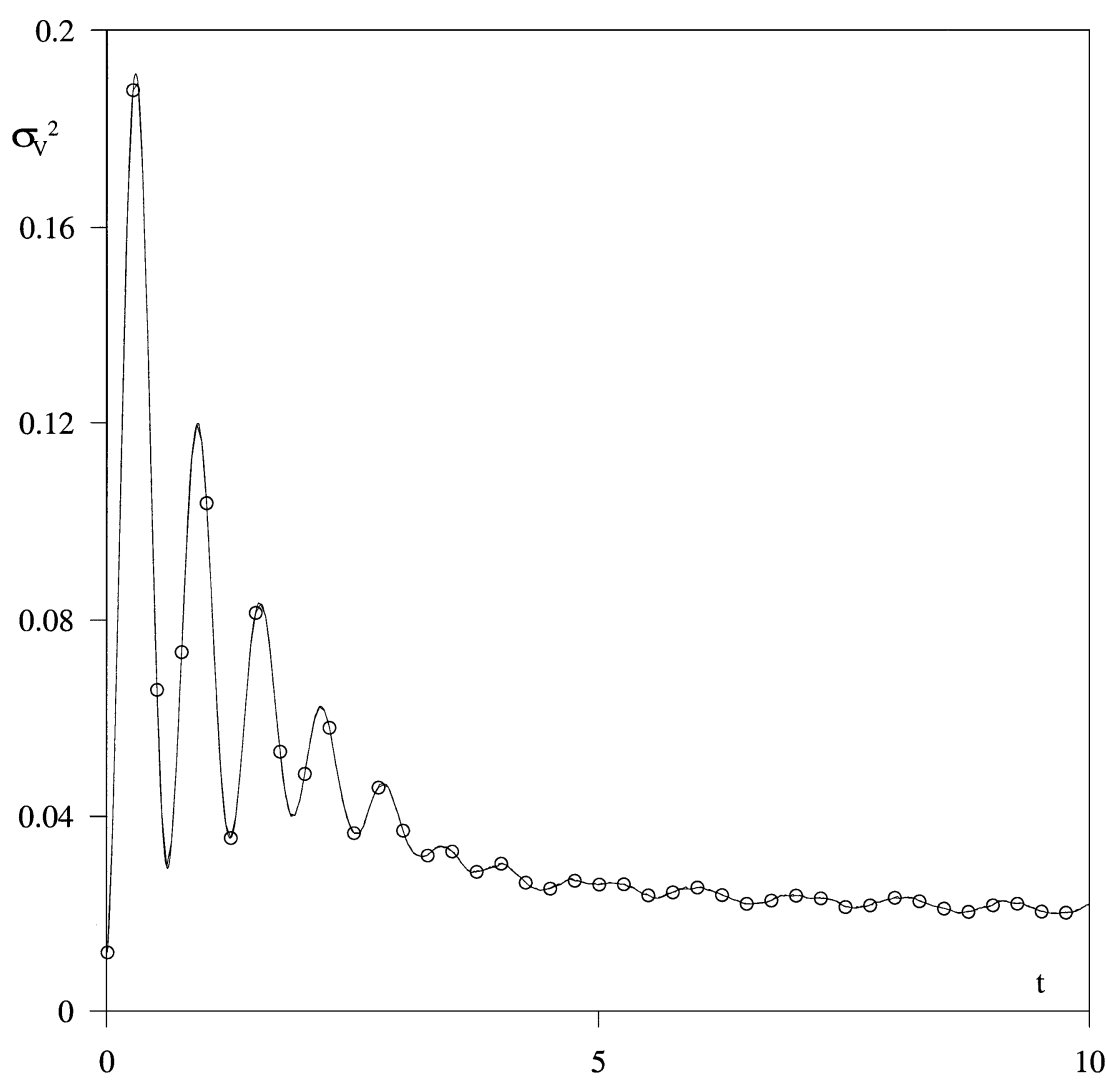

Figure 5. Comparison between analytical solution and simulation for the velocity variance, continuous line $-\sigma_{V}^{2}$, dotted line - simulation.

subject to the following initial conditions

$$
\left\{\begin{array}{l}
\tau(0)=0 \\
y(0)=y^{\prime} \\
\dot{y}(0)=\dot{y}^{\prime} \\
p(0)=p_{t}\left(y^{\prime}, \dot{y}^{\prime} ; t\right) .
\end{array}\right.
$$

Solution of Equation (30) takes the form

$$
\left\{\begin{array}{l}
\tau=s+C_{1} \\
y(s)=C_{2} e^{-D \omega_{1} s} \cos \left(\bar{\omega}_{1} s\right)+C_{3} e^{-D \omega_{1} s} \sin \left(\bar{\omega}_{1} s\right)+y_{p}(s) \\
p(s)=p(0) \exp (c s)+G_{t}(y, \dot{y} ; t) \tau
\end{array}\right.
$$

where

$$
\bar{\omega}_{1}=\omega_{1} \sqrt{1-D^{2}}, \quad c=2 D \omega_{1}-\lambda
$$




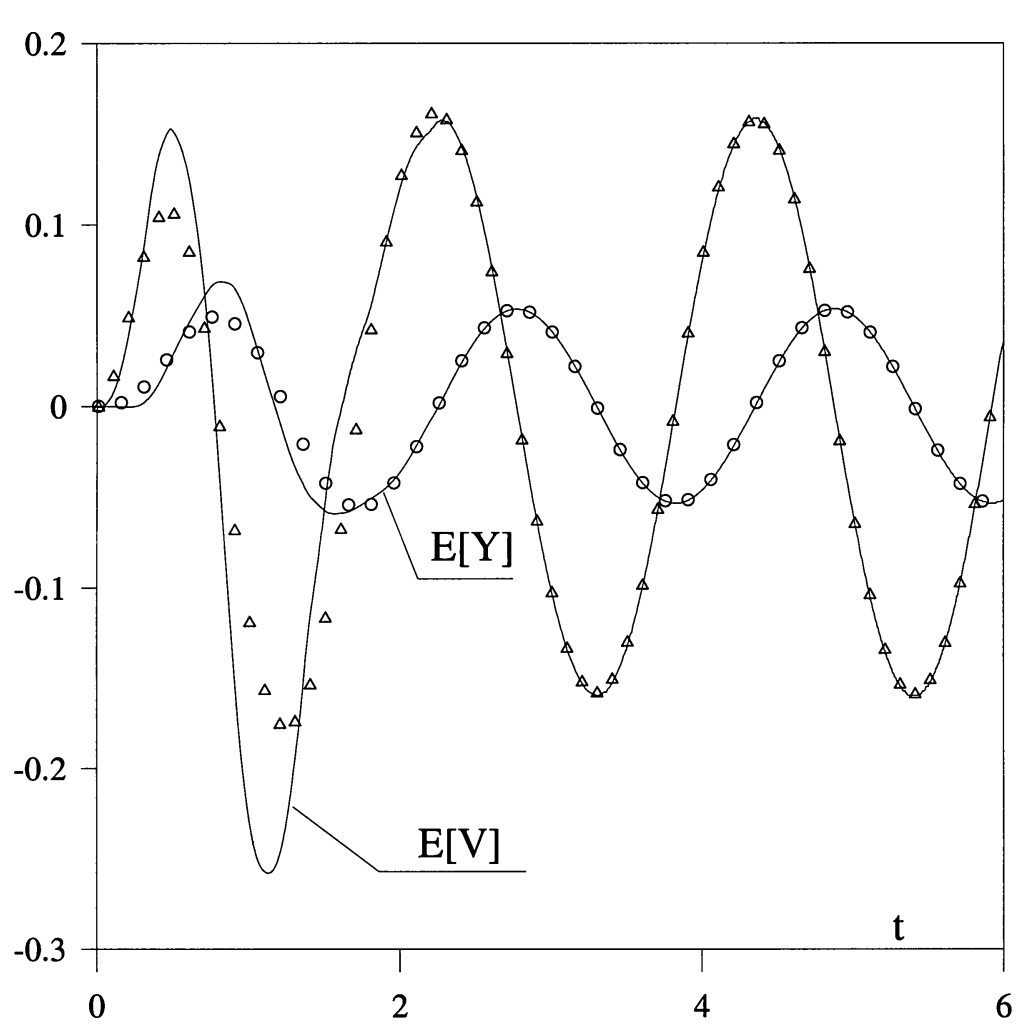

Figure 6. Comparison between analytical solution and simulation in the nonlinear case for the mean of displacement and velocity. Continuous line $-E[X]$, dashed line $-E[V]$, dotted line - simulation.

and $y_{p}(s)$ is the known particular solution of Equation (30) The solution found is finally again

$$
p(y, \dot{y}, t+\tau)=e^{c \tau} p_{t}\left(y^{\prime}(y, \dot{y}), \dot{y}^{\prime}(y, \dot{y}) ; t\right)+G_{t}(y, \dot{y} ; t) \tau
$$

where $y^{\prime}(y, \dot{y})$ and $\dot{y}^{\prime}(y, \dot{y})$ can be explicitly obtained from Equations (31) and (32).

\section{Nonlinear Oscillations of a Beam Under Transversal and Axial Poisson Pulses}

Let us now consider the case in which a simply supported beam is subjected to a train of stationary axial Poisson pulses and external harmonic loading and presents a nonlinear behavior defined by [19]

$$
E J \frac{\partial^{4} w}{\partial x^{4}}+\mu \frac{\partial^{2} w}{\partial t^{2}}+\rho \frac{\partial w}{\partial t}+P \xi(t) \frac{\partial^{2} w}{\partial x^{2}}+\frac{1}{2 l} \frac{\partial^{2} w}{\partial x^{2}} \int_{0}^{l}\left(\frac{\partial w}{\partial x}\right)^{2} d x=\sin (\Omega t) .
$$

Again we express the solution as an expansion in terms of the linear free oscillation modes

$$
w(x, t)=\sum_{n=1}^{\infty} \phi_{n}(x) Y_{n}(t)
$$




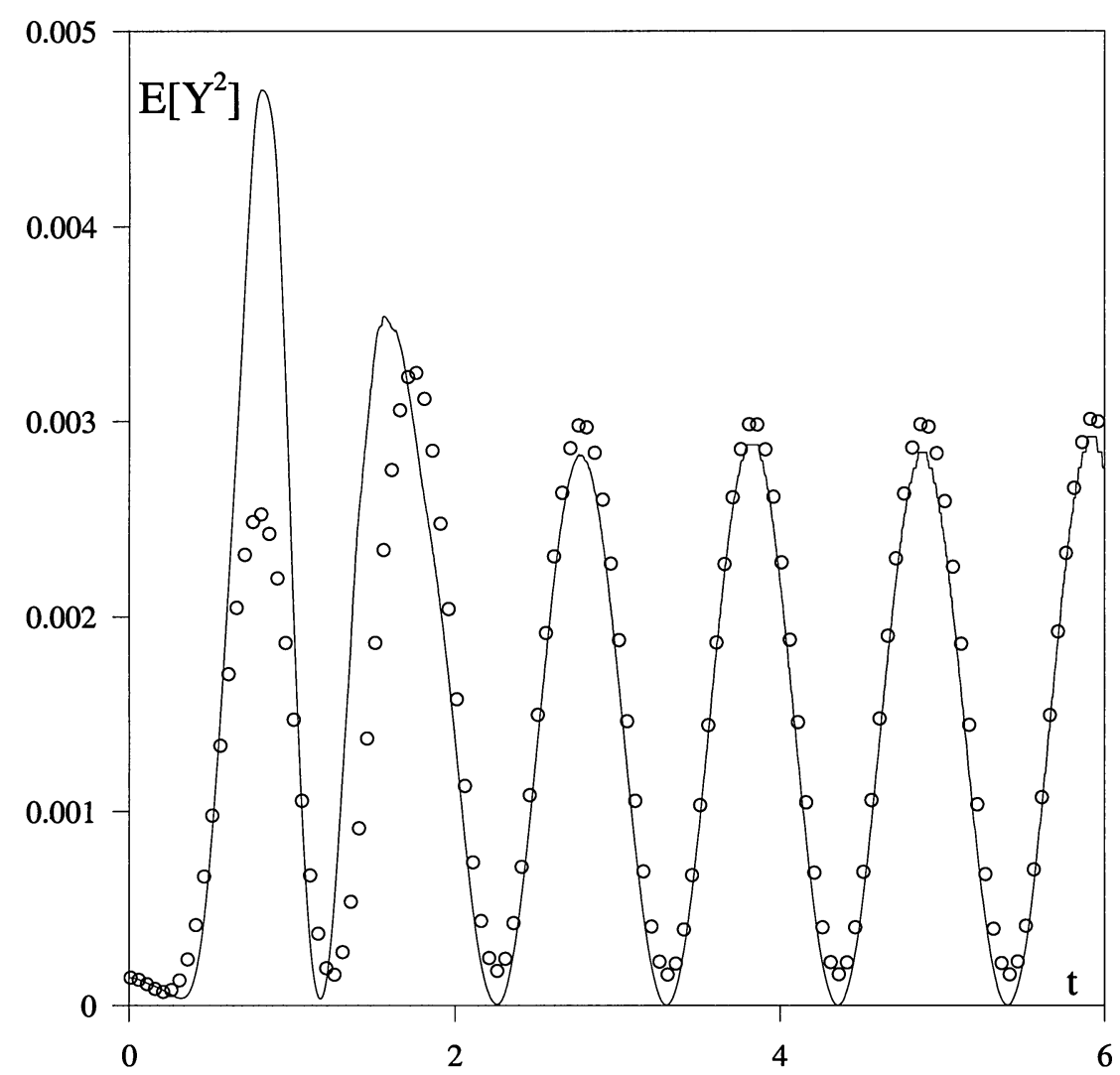

Figure 7. Comparison between analytical solution and simulation in the nonlinear case for the mean square of displacement. Continuous line $-E\left[X^{2}\right]$, dotted line - simulation.

and using the well-known orthogonality properties of the eigenfunction basis, the following set of coupled nonlinear equations is obtained:

$$
\ddot{Y}_{n}+2 D \omega_{n} \dot{y}_{n}+\omega_{n}^{2}\left[1-\varepsilon_{n} \xi(t)\right] Y_{n}+\sum_{m, p, q=1=0}^{\infty} \Gamma_{n m p q} Y_{m} Y_{p} Y_{q}=P_{n} \sin (\Omega t), \quad n=1,2, \ldots
$$

in which

$$
\Gamma_{n m p q}=-\frac{1}{2 l}\left[\int_{0}^{l} \phi_{n}^{\prime} \phi_{m}^{\prime} d x\right]\left[\int_{0}^{l} \phi_{p}^{\prime} \phi_{q}^{\prime} d x\right]
$$

For the simple case in which $n=m=p=q=1$, the following parametric, nonlinear stochastic differential equation is obtained

$$
\ddot{Y}+2 D \omega_{1} \dot{y}+\omega_{1}^{2}\left[1-\varepsilon_{1} \xi(t)\right] Y+\gamma Y^{3}=\sin (\Omega t)
$$

where $\gamma=\Gamma_{1111}$. The probabilistic characterization of the response can be achieved, when truncating at the first mode, by considering the well-known Kolmogorov-Feller integro-differential equation 


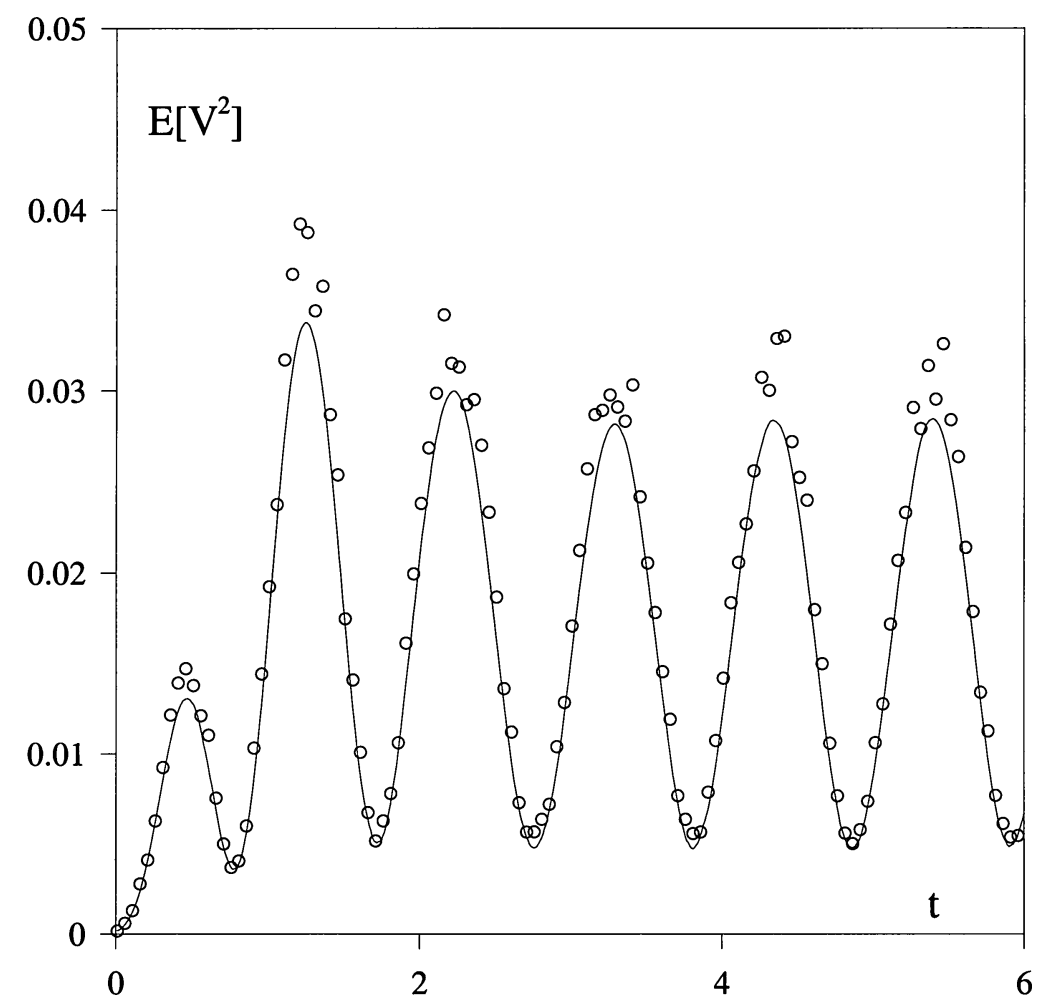

Figure 8. Comparison between analytical solution and simulation in the nonlinear case for the mean square of velocity. Continuous line $-E\left[V^{2}\right]$, dotted line - simulation.

$$
\begin{aligned}
\frac{\partial p}{\partial \tau} & =2 D \omega_{1} \frac{\partial(\dot{y} p)}{\partial \dot{y}}+\left(\omega_{1}^{2} y+\gamma y^{3}\right) \frac{\partial p}{\partial \dot{y}}-\dot{y} \frac{\partial p}{\partial y} \\
& +\lambda \int_{\mathrm{a}} p\left(y, \dot{y}-\omega_{1}^{2} \varepsilon y a ; t+\tau / y^{\prime}, \dot{y}^{\prime} ; t\right) p_{A}(a) d a-\lambda p .
\end{aligned}
$$

Using the same approach outlined for the linear case, the following differential equation for the joint probability density function $p_{t+\tau}=p(y, \dot{y} ; t+\tau)$ is obtained

$$
\frac{\partial p_{t+\tau}}{\partial \tau}=2 D \omega_{1} \frac{\partial\left(\dot{y} p_{t+\tau}\right)}{\partial \dot{y}}+\left(\omega_{1}^{2} y+\gamma y^{3}+\sin (\Omega t)\right) \frac{\partial p_{t+\tau}}{\partial \dot{y}}-\dot{y} \frac{\partial p_{t+\tau}}{\partial y}+\lambda G_{t}(y, \dot{y} ; t)-\lambda p_{t+\tau} .
$$

To construct the solution surface by the Method of Characteristics one solves the following system of ordinary differential equations on $s$ :

$$
\left\{\begin{array}{l}
\frac{d \tau}{d s}=1 \\
\frac{d y}{d s}=\dot{y} \\
\frac{d \dot{y}}{d s}=-\left(2 D \omega_{1} \dot{y}+\omega_{1}^{2} y+\gamma y^{3}+\sin (\Omega t)\right) \\
\frac{d p}{d s}=G_{t}(y, \dot{y} ; t)+\left(2 D \omega_{1}-\lambda\right) p
\end{array}\right.
$$




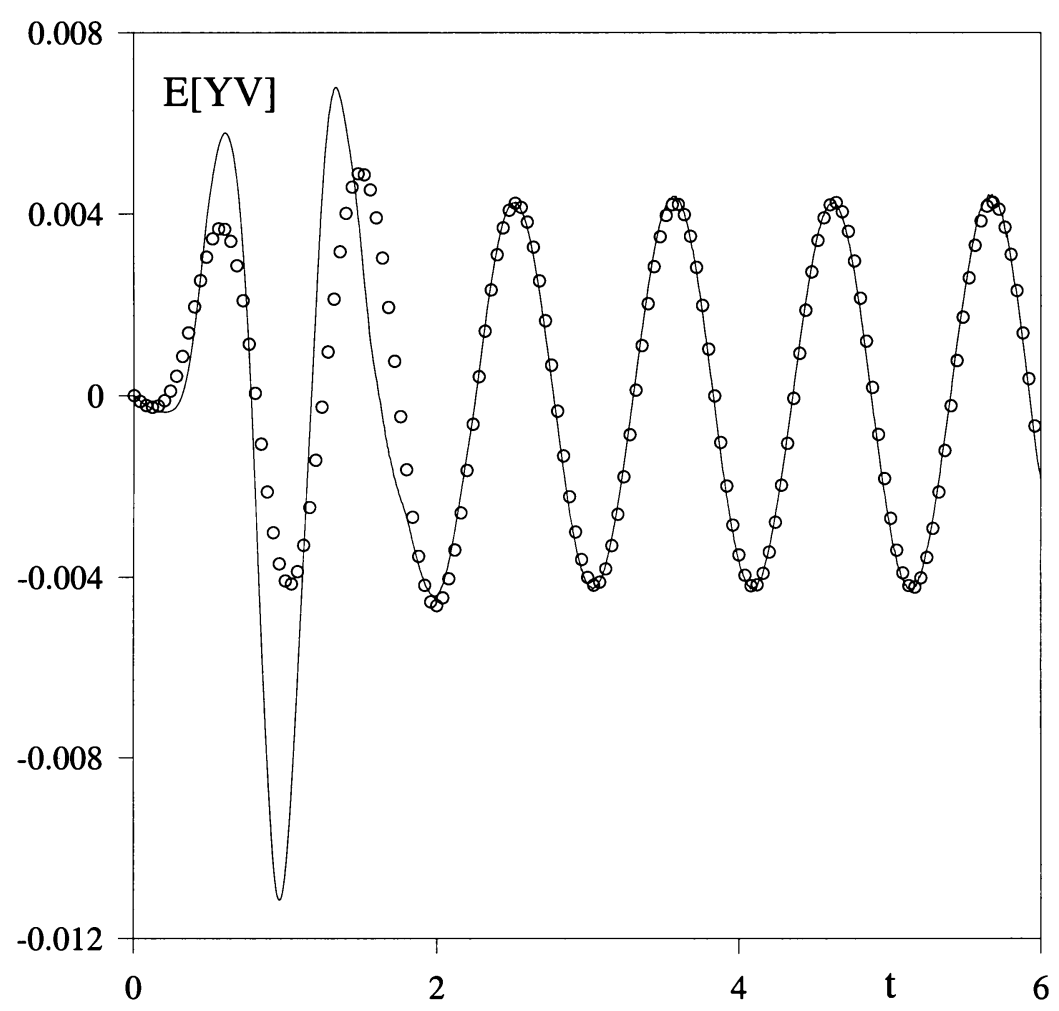

Figure 9. Comparison between analytical solution and simulation in the nonlinear case for the cross mean square of displacement velocity. Continuous line $-E[X V]$, dotted line - simulation.

where

$$
G_{t}(y, \dot{y} ; t)=\lambda \int_{\mathrm{a}} p\left(y, \dot{y}-\omega_{1}^{2} \varepsilon y a ; t\right) p_{A}(a) d a
$$

subject to the following initial conditions

$$
\left\{\begin{array}{l}
\tau(0)=0 \\
y(0)=y^{\prime} \\
\dot{y}(0)=\dot{y}^{\prime} \\
p(0)=p_{t}\left(y^{\prime}, \dot{y}^{\prime} ; t\right) .
\end{array}\right.
$$

The solution $p(y, \dot{y}, t+\tau)$ can be written as follows:

$$
p(y, \dot{y}, t+\tau)=e^{c \tau} p_{t}\left(y^{\prime}(y, \dot{y}), \dot{y}^{\prime}(y, \dot{y}) ; t\right)+G_{t}(y, \dot{y} ; t) \tau
$$

It should be noted that in this case the nonlinear behavior of the beam does not allow construction of an explicit solution for the characteristic curves and only numerical integration can be pursued. 


\section{Applications}

A cell-to-cell mapping numerical procedure has been used to describe the features of the analytical solution found in Equation (20) for the analysis of a linear beam under transversal Poisson pulses. A simply supported steel beam has been considered, having dimensions $b \times h=7 \times 200 \mathrm{~mm}$, length $l=5 \mathrm{~m}$ and damping ratios $D=0.01$. The initial distribution $p_{0}(y, \dot{y}, 0)$ has been assumed to be a zero-mean Gaussian distribution with variances $\sigma_{Y}^{2}=1$ and $\sigma_{\dot{y}}^{2}=1$. The Poisson train pulses considered have mean arrival rate $\lambda=10$ and zero-mean Gaussian distribution with intensity variance $\sigma_{a}^{2}=0.5$. The time integration step used for the numerical integration has been set as $\Delta t=0.001 \mathrm{~s}$ and the mesh grid has size $\Delta y=0.02 \mathrm{~mm}$ and $\Delta \dot{y}=0.02 \mathrm{~mm}$. Figures 1 and 2 show the transition to the stationary solution $(t=0,3,6 \mathrm{~s})$ for the marginal displacement and velocity distribution compared to Monte Carlo simulation solution $(v=\dot{y})$. The results obtained confirm that the analytical solution described above is highly effective when compared to simulation techniques.

The effect of an axial Poisson train of pulses on a linear beam has also been investigated, Equation (34), for a simply supported steel beam with damping ratios $D=0.1$, while $\Omega=3 \mathrm{rad} / \mathrm{s}$. The initial distribution $p_{0}(y, \dot{y}, 0)$ has been assumed as a zero-mean Gaussian distribution with variances $\sigma_{Y}^{2}=0.1$ and $\sigma_{\dot{y}}^{2}=0.1$. Poisson train pulses are considered with mean arrival rate $\lambda=5$ and zero-mean Gaussian distribution with intensity variance $\sigma_{a}^{2}=0.5$. The time integration step used for the numerical integration has been set as $\Delta t=0.001 \mathrm{~s}$ and the mesh grid has size $\Delta y=0.02 \mathrm{~mm}$ and $\Delta \dot{y}=0.02 \mathrm{~mm}$. Figures 3-5 show the time evolutionary mean and variance of displacement and velocity, respectively, compared to Monte Carlo simulation.

Finally, the effect of an axial Poisson train of pulses on a beam having nonlinear behavior has been investigated, Equation (45), on a simply supported beam with damping ratios $D=0.3, \Omega=3 \mathrm{rad} / \mathrm{s}$ and nonlinear restoring force coefficient $\gamma=200$. The initial distribution $p_{0}(y, \dot{y}, 0)$ has been assumed as a zero-mean Gaussian distribution with variances $\sigma_{Y}=0.012$ and $\sigma_{\dot{y}}=0.012$. The Poisson train pulses considered have mean arrival rate $\lambda=10$ and zero-mean Gaussian distribution with intensity variance $\sigma_{a}=0.5$. The time integration step used for the numerical integration has been set as $\Delta t=0.001 \mathrm{~s}$ and the mesh grid has size $\Delta y=0.0015 \mathrm{~mm}$ and $\Delta \dot{y}=0.002 \mathrm{~mm}$. Figures 6-9 show the time evolutionary mean and variance of displacement and velocity, respectively, compared to Monte Carlo simulation. The evaluation of the characteristic curves, Equation (42), has been obtained through numerical integration by a Runge-Kutta fourth-order method. Comparison with the statistics obtained in the quasilinear case (Figures 6-9) shows that the nonlinear effect is effective, especially in the evaluation of the nonstationary solution, while the stationary solution is obtained with good accuracy. This is due to the knowledge, in the quasilinear case, of a closed-form solution of the characteristic curves, while only a numerical solution can be pursued in the nonlinear case.

\section{Conclusions}

An approximate explicit response probability density function of a beam under external and axial impulsive random Poisson excitation has been obtained as approximate solution of the Kolmogorov-Feller equation. The integro-differential Kolmogorov-Feller equation has been reduced to a first-order partial differential equation, using physical considerations about the behavior of the response for small values of the time step. The well-known Method of Characteristics has then been applied to obtain the required solution. The correctness of the solution has been validated through Monte Carlo simulation of a linear and a nonlinear beam driven by Poisson external and parametric pulses. 


\section{Acknowledgments}

The authors wish to thank Rodolfo Repetto and Corrado Lattanzio for discussions on mathematical and computational topics related to the subject of this paper.

\section{References}

1. Lin, Y. K., 'Application of non stationary shot noise in the study of system response to a class of non-stationary excitations', Journal of Applied Mechanics 30, 1963, 555-558.

2. Lin, Y. K. and Cai, G. Q., Probabilistic Structural Dynamics: Advanced Theory and Applications, McGraw-Hill, New York, 1995.

3. Tung, C. C., 'Random response of highway bridges to vehicle loads', ASCE Journal of Engineering and Mechanical Division 93, 1967, 79-94.

4. Gihman, I. I. and Skorohod, A.V., Stochastic Differential Equations, Springer Verlag, Berlin, 1972.

5. Di Paola, M. and Falsone, G., 'Stochastic dynamics of non-linear systems driven by non-normal delta-correlated processes', Journal of Applied Mechanics 60, 1993, 141-148.

6. Gardiner, C. W., Handbook of Stochastic Methods, Springer-Verlag, Berlin, 1990.

7. Risken, H., The Fokker-Planck Equation: Methods of Solution and Applications, Springer-Verlag, Berlin, 1984.

8. Feng, G. M., Wang, B., and Lu, Y. F., 'Path integral, functional method and stochastic dynamical systems', Probabilistic Engineering Mechanics 7, 1992, 149-157.

9. Naess, A. and Johnses, J. M., 'Response statistics of nonlinear, compliant offshore structures by the path integral solution method', Probabilistic Engineering Mechanics 8, 1993, 91-106.

10. Di Paola, M. and Vasta, M., 'Stochastic integro-differential and differential equations of non linear systems excited by parametric Poisson Pulses', International Journal of Non-Linear Mechanics 31, 1997, 855-862.

11. Vasta, M., 'Exact stationary solution for a class of non-linear systems driven by a non-normal delta-correlated process', International Journal of Non-Linear Mechanics 30, 1995, 407-418.

12. Proppe, C., 'Exact stationary probability density functions for non-linear systems under Poisson white noise excitation', International Journal of Non-Linear Mechanics 38, 2003, 557-564.

13. Koyluoglu, H. U., Nielsen, R. K., and Iwankievicz, R., 'Response and reliability of Poisson-driven systems by path integration', Journal of Engineering Mechanics 1, 1995, 117-130.

14. Iwankievicz, R. and Nielsen, S. R. K., 'Dynamic response of non-linear systems to Poisson distribuited random impulses', Journal of Sound and Vibration 156, 1992, 407-423.

15. Roberts, J. B., 'System response to random impulses', Journal of Sound and Vibration 24, 1972, $23-34$.

16. Vasta, M. and Roberts, J. B., 'An approximate transition probability density function for non-linear systems to impulsive loads', in Proceedings of the Third International Conference on Computational Stochastic Mechanics, P. D. Spanos (ed.), Santorini, Greece, Balkema, Rotterdam, The Netherlands, 1999.

17. Guenther, R. B. and Lee, J.W., Partial Differential Equations of Mathematical Physics and Integral Equations, Prentice Hall, Upper Saddle River, New Jersey, 1988.

18. Renger, A., 'Eine dichtegleichung fur schwingungssysteme bei gleichzeitigen kontinuierlichen und diskreten stochastischen erregungen', ZAMM 59, 1979, 1-13.

19. Nayfeh, A. H. and Balachandran, B., Applied Nonlinear Dynamics, Wiley, New York, 1995. 